\title{
Modeling Noisiness to Recognize Named Entities using Multitask Neural Networks on Social Media
}

\author{
Gustavo Aguilar ${ }^{\star}$ A. Pastor López-Monroy ${ }^{\star}$ Fabio A. González ${ }^{\dagger}$ Thamar Solorio $^{\star}$ \\ ${ }^{\star}$ Department of Computer Science, University of Houston \\ ${ }^{\dagger}$ Systems and Computer Engineering Department, Universidad Nacional de Colombia \\ \{gaguilaralas, alopezmonroy\}@uh.edu \\ fagonzalezo@unal.edu.co, solorio@cs.uh.edu
}

\begin{abstract}
Recognizing named entities in a document is a key task in many NLP applications. Although current state-of-the-art approaches to this task reach a high performance on clean text (e.g. newswire genres), those algorithms dramatically degrade when they are moved to noisy environments such as social media domains. We present two systems that address the challenges of processing social media data using character-level phonetics and phonology, word embeddings, and Part-of-Speech tags as features. The first model is a multitask end-toend Bidirectional Long Short-Term Memory (BLSTM)-Conditional Random Field (CRF) network whose output layer contains two CRF classifiers. The second model uses a multitask BLSTM network as feature extractor that transfers the learning to a CRF classifier for the final prediction. Our systems outperform the current F1 scores of the state of the art on the Workshop on Noisy User-generated Text 2017 dataset by $2.45 \%$ and $3.69 \%$, establishing a more suitable approach for social media environments.
\end{abstract}

\section{Introduction}

One of the core tasks in Natural Language Processing (NLP) is Named Entity Recognition (NER). NER is a sequence tagging task that consists in selecting the words that describe entities and recognizing their types (e.g., a person, location, company, etc.). Figure 1 shows examples of sentences from different domains that contain named entities. Recognizing entities in running text is typically one of the first tasks in the pipeline of many NLP applications, including machine translation, summarization, sentiment analysis, and question answering.

Traditional machine learning systems have proven to be effective in formal text, where grammatical errors are minimal and writers stick to

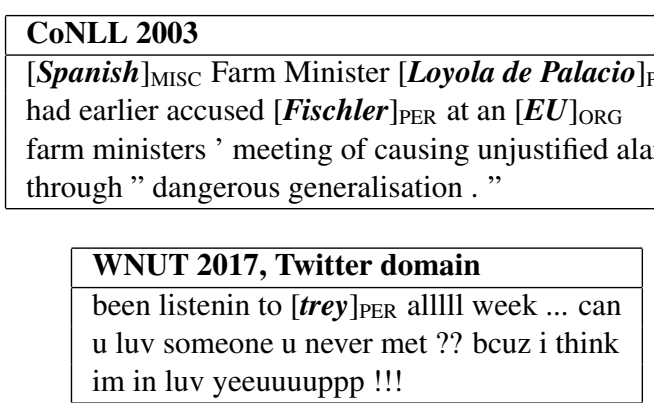

Figure 1: Examples from the CoNLL 2003 and the WNUT 2017 datasets. The noise from the WNUT dataset makes a clear difference from one text to the other, establishing new challenges to the current stateof-the-art systems on formal text. The words in bold are grouped to described the entities.

the rules of the written language (Florian et al., 2003a; Chieu and Ng, 2003a). However, those traditional systems dramatically fail on informal text, where improper grammatical structures, spelling inconsistencies, and slang vocabulary prevail (Ritter et al., 2011). For instance, Table 1 shows a snapshot of NER systems' performance during the last years, where the results drop from $96.49 \%$ to $41.86 \%$ on the F1 metric as we move from formal to informal text. Although the results are not directly comparable because they consider different conditions and challenges, they serve as strong evidence that the NER task in social media is far from being solved.

Recently, researchers have approached NER using different neural network architectures. For instance, Chiu and Nichols (2016) proposed a neural model using Convolutional Neural Networks $(\mathrm{CNN})$ for characters and a bidirectional Long Short Term Memory (LSTM) for words. Their model learned from word embeddings, capitalization, and lexicon features. On a slightly different approach, Lample et al. (2016) used a BLSTM with a CRF at the output layer, re- 


\begin{tabular}{lllll}
\hline Organizer & Competition & Domain & F1 & Classes \\
\hline Grishman and Sundheim (1996a) & MUC-6 & Newswire & $96.49 \%$ & 2 \\
Tjong Kim Sang and De Meulder (2003) & CoNLL & Newswire & $88.76 \%$ & 4 \\
Strauss et al. (2016) & WNUT & Twitter & $52.41 \%$ & 10 \\
Derczynski et al. (2017) & WNUT & SM domains & $41.86 \%$ & 6 \\
\hline
\end{tabular}

Table 1: Results on different NER shared tasks. The performance degrades as the systems are moved to social media (SM) environments. The last row considers multiple SM domains, such as Twitter, YouTube, Reddit, and StackExchange.

moving the dependencies on external resources. Moreover, Ma and Hovy (2016) proposed an end-to-end BLSTM-CNN-CRF network, whose loss function is based on the maximum loglikelihood estimation of the CRF. These architectures were benchmarked on the standard CoNLL 2003 dataset (Tjong Kim Sang and De Meulder, 2003). Although most of the work has focused on formal datasets, similar approaches have been evaluated on SM domains (Strauss et al., 2016; Derczynski et al., 2017). In the Workshop on Noisy User-generated Text (WNUT) 2016, Limsopatham and Collier (2016), the winners of the NER shared task, used a BLSTM-CRF model that induced features from an orthographic representation of the text. Later, in the WNUT 2017 shared task, the best performing system used a multitask network that transferred the learning to a CRF classifier for the final prediction (Aguilar et al., 2017).

In this work we focus on addressing the challenges of the NER task found in social media environments. We propose that what is traditionally categorized as noise (i.e., misspellings, inconsistent orthography, emerging abbreviations, and slang) should be modeled as is since it is an inherent characteristic of SM text. Specifically, the proposed models attempt to address i) misspellings using subword level representations, ii) grammatical mistakes with SM-oriented Part-ofSpeech tags (Owoputi et al., 2013), iii) sounddriven text with phonetic and phonological features (Bharadwaj et al., 2016), and iv) the intrinsic skewness of NER datasets by applying class weights. It is worth noting that our models do not rely on capitalization or any external resources such as gazetteers. The reasons are that capitalization is arbitrarily used on SM environments, and gazetteers are expensive resources to develop for a scenario where novel entities constantly and rapidly emerge (Derczynski et al., 2017; Augenstein et al., 2017).
Based on our experiments, we have seen that a multitask variation of the proposed networks improves the results over a single-task network. Additionally, this multitask version, paired with phonetic and phonological features, outperforms previous state-of-the-art results on the WNUT 2017 dataset, and the same models obtain reasonable results with respect to the state of the art on the CoNLL 2003 dataset (Tjong Kim Sang and De Meulder, 2003).

The rest of the paper is organized as follows: $\S 2$ presents the proposed features, the formal description of the models, and the implementation details. $\S 3$ describes the datasets and their challenges. On $\S 4$, we show the evaluation process of our models and the results. We explain the performance of the models on $\S 5$. $\S 6$ describes related work and, finally, we draw conclusions on $\S 7$.

\section{Methods}

Our methods are based on two main strategies: i) a representation of the input text using complementary features that are more suitable to social media environments, and ii) a fusion of these features by using a multitask neural network model whose main goal is to learn how entities are contextualized with and without the entity type information.

\subsection{Feature representation}

Semantic features. Semantic features play a crucial role in our pipeline as they provide contextual information to the model. This information allows the model to infer the presence of entities as well as the entity types. We use the pretrained word embedding model provided by Godin et al. (2015). This model has been trained on 1 million tweets (roughly $1 \%$ of the tweets in a year) with the skipgram algorithm. We take advantage of this resource as it easily adapts to other SM environments besides Twitter (Aguilar et al., 2017).

Syntactic features. Syntactic features help the models deal with word disambiguation based on 


\begin{tabular}{|l|l|}
\hline Sentence & IPA \\
\hline $\begin{array}{l}\text { u hav to b KIDDDDING me } \\
\text { you have to be kidding me }\end{array}$ & $\begin{array}{l}\text { /ju hæv to bi kıdıj mi// } \\
\text { /ju hæv to bi kıdıj mi// }\end{array}$ \\
\hline
\end{tabular}

Table 2: Examples of both noisy and normalized text. In both cases, the mappings to the International Phonetic Alphabet (IPA) are the same.

the grammatical role that the words play on a sentence. That is, a word that can be a verb or a noun in different scenarios may conflict with the interpretations of the models; however, by providing syntactical information the models can improve their decisions. We capture grammatical patterns using the Part-of-Speech (POS) tagger provided by Owoputi et al. (2013). This POS tagger has custom labels that are suitable to SM data (i.e., the tagger considers emojis, hashtags, URLs and others).

Phonetic and phonological features. We also consider the phonetic and phonological aspects of the data at the character level. In Table 2 we show an example of two phrases: the first sentence is taken from SM, and the second one is its normalized representation. Even though the spellings of both phrases are significantly different, by using the phonological (articulatory) aspects of those phrases it is possible to map them to the same phonetic representation. In other words, our assumption is that social media writers heavily rely on the way that words sound while they write. We use the Epitran ${ }^{1}$ library (Bharadwaj et al., 2016), which transliterates graphemes to phonemes with the International Phonetic Alphabet (IPA). In addition to the IPA phonemes, we also use the phonological (articulatory) features generated by the PanPhon ${ }^{2}$ library (Mortensen et al., 2016). These features provide articulatory information such as the way the mouth and nasal areas are involved in the elaboration of sounds while people speak.

\subsection{Models}

We have experimented with two models. In the first one, we use an end-to-end BLSTM-CRF network with a multitask output layer comprised of one CRF per task, similar to Yang et al. (2016). In the second one, we define a stacked model that is based on two phases: i) a multitask neural network and ii) a CRF classifier. In the first phase, the network acts as a feature extractor, and then,

\footnotetext{
${ }^{1}$ https://github.com/dmort27/epitran

${ }^{2}$ https://github.com/dmort27/panphon
}

for the second phase, it transfers the learning to a CRF classifier for the final predictions (see Figure 3). In both cases, the multitask layer is defined with the following two tasks:

- Segmentation. This task focuses on the Begin-Inside-Outside (BIO scheme) level of the tokens. That is, for a given NE, the model has to predict whether a word is B, I, or O regardless of the entity type. The idea is to let the models learn how entities are treated in general, rather than associating the types to certain contexts. This task acts as a regularizer of the primary task to prevent overfitting.

- Categorization. In this case, the models have to predict the types of the entities along with the BIO scheme (e.g., B-person, Iperson, etc.), which represent the final labels.

We formalize the definitions of our models as follows: let $X=\left[x_{1}, x_{2}, \ldots, x_{n}\right]$ be a sample sentence where $x_{i}$ is the $i^{t h}$ word in the sequence. Then, let $\alpha: V_{x} \rightarrow \mathbb{R}^{\operatorname{dim}_{x}}$ be a word embedding, and let $\mathbf{x}=\left[\alpha\left(x_{1}\right), \ldots, \alpha\left(x_{n}\right)\right]$ be the word embedding matrix for the sample sentence such that $V_{x}$ is the vocabulary and $\operatorname{dim}_{x}$ is the dimension of the embedding space. Similarly, let $\beta: V_{p} \rightarrow \mathbb{R}^{\operatorname{dim}_{p}}$ be the POS tag embedding, and let $\mathbf{p}=\left[\beta\left(p_{1}\right), \ldots, \beta\left(p_{n}\right)\right]$ be the POS tag embedding matrix for the sample sentence such that $V_{p}$ is the set of Part-of-Speech tags and $\operatorname{dim}_{p}$ is the dimension of the embedding space. Notice that the POS tag embedding matrix $\mathbf{p}$ is learned during training. Also, let $Q=\left[q_{1}, q_{2}, \ldots, q_{m}\right]$ be the phonetic letters of a word; let $\gamma: V_{q} \rightarrow$ $\mathbb{R}^{\left|V_{q}\right|+\text { dim }_{\text {PanPhon }}}$ be an embedding that maps each phonetic character to a one-hot vector of the International Phonetic Alphabet $\left(V_{q}\right)$ concatenated with the 21 (dim $\left._{\text {PanPhon }}\right)$ phonological features of the PanPhon library (tongue position, movement of lips, etc.) (Bharadwaj et al., 2016); and let $\mathbf{q}=\left[\gamma\left(q_{1}\right), \ldots, \gamma\left(q_{m}\right)\right]$ be the matrix representation of the word-level phonetics and phonology.

We first apply an LSTM (Hochreiter and Schmidhuber, 1997) to the q matrix on forward and backward directions. Then we concatenate the output from both directions:

$$
\begin{aligned}
\overrightarrow{\mathbf{h}} & =\operatorname{LSTM}\left(\left\{\mathbf{q}_{1}, \mathbf{q}_{2}, \ldots, \mathbf{q}_{m}\right\}\right) \\
\overleftarrow{\mathbf{h}} & =\operatorname{LSTM}\left(\left\{\mathbf{q}_{m}, \mathbf{q}_{m-1}, \ldots, \mathbf{q}_{1}\right\}\right) \\
\mathbf{h} & =[\overrightarrow{\mathbf{h}} ; \overleftarrow{\mathbf{h}}]
\end{aligned}
$$




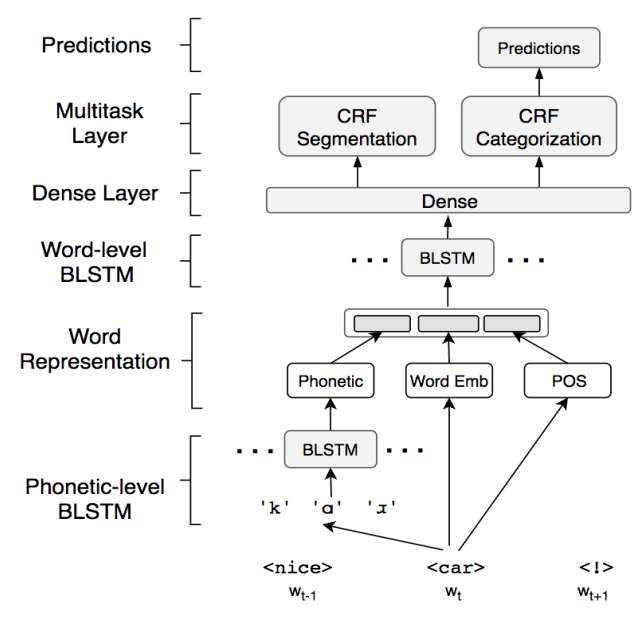

Figure 2: This is an end-to-end system that uses the CRF loss function as the objective function of the network. It also uses multitask learning on the output layer.

This vector not only encodes the phonetic and phonological features, but it also captures some morphological patterns at the character level based on the IPA representations. Then, we concatenate this vector with the word and POS tag representations: $\mathbf{a}=\left[\mathbf{x}_{t} ; \mathbf{p}_{t} ; \mathbf{h}_{t}\right]$. We feed this representation to another bidirectional LSTM network (Dyer et al., 2015), similar to the BLSTM described for the character level. The bidirectional LSTM generates a word-level representation that accounts for the context in the sentence using semantics, syntax, phonetics and phonological aspects. We feed this representation to a fully-connected layer:

$$
\begin{gathered}
\mathbf{r}_{i}=\operatorname{BLSTM}\left(\left\{\mathbf{a}_{1}, \mathbf{a}_{2}, \ldots \mathbf{a}_{n}\right\}\right) \\
\mathbf{z}_{i}=\operatorname{ReLU}\left(\mathbf{W}_{a} \mathbf{r}_{i}+\mathbf{b}\right)
\end{gathered}
$$

At this point, both models share the same definition. From here, we describe the multitask learning characteristics for each model separately.

End-to-end model. For the end-to-end network (see Figure 2), we define an output layer based on two Conditional Random Fields (Lafferty et al., 2001), each assigned to one of the tasks. The idea of adding a CRF to the model is to capture the relation of the output probabilities of the network with respect to the whole sequence. This means that the CRFs will maximize the log-likelihood of the entire sequence, which allows the model to learn very specific constraints from the data (e.g., a label I-location cannot be followed by $I$ person). Following Ma and Hovy (2016), we formalize the definition of the CRF as follows: let $\mathbf{y}=\left[y_{1}, y_{2}, \ldots, y_{n}\right]$ be the labels for a sequence $\mathbf{x}$,

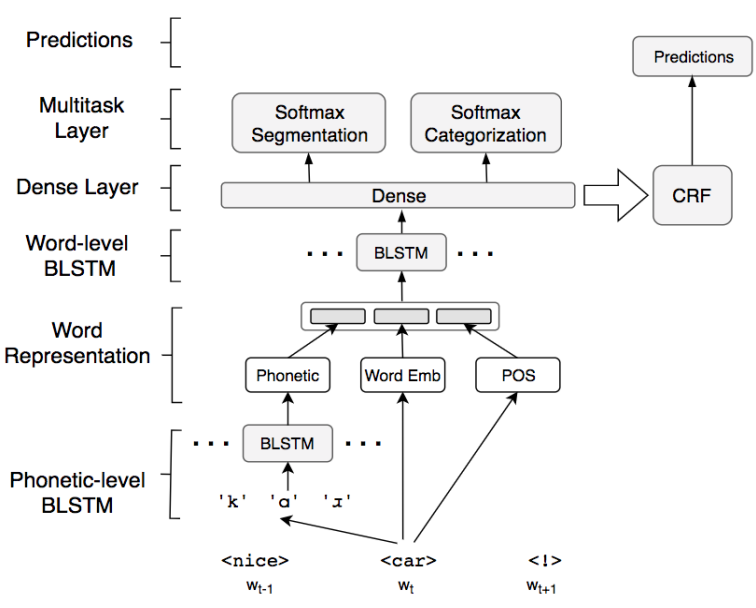

Figure 3: This is a stacked model that uses a network as feature extractor, and then it transfers the learning to a CRF classifier. The network uses multitask learning to capture the features.

where $y_{i}$ represents the $i^{\text {th }}$ label of the $\mathbf{x}_{i}$ token in the sentence. Next, we calculate the conditional probability of seeing $\mathbf{y}$ given the extracted features $\mathbf{z}$ from the network and the weights $\mathbf{W}$ associated to the labels:

$$
p(\mathbf{y} \mid \mathbf{z} ; \mathbf{W})=\frac{\exp \left(\mathbf{W}_{y} \Phi(\mathbf{z}, \mathbf{y})\right)}{\sum_{\mathbf{y}^{\prime} \in \mathbf{y}} \exp \left(\mathbf{W}_{y} \Phi\left(\mathbf{z}, \mathbf{y}^{\prime}\right)\right)}
$$

Where $\Phi$ is a feature function that codifies the interactions between consecutive labels, $y_{t}$ and $y_{t+1}$, as well as the interactions between labels and words, represented by $z_{t}$. Then, the objective function for one CRF is defined by the maximum log-likelihood of this probability. However, we are running two CRFs as the objective function:

$$
\begin{aligned}
\mathcal{L}_{1}(\mathbf{z}, \mathbf{W}) & =\log p\left(\mathbf{y}_{\text {seg }} \mid \mathbf{z} ; \mathbf{W}\right) \\
\mathcal{L}_{2}(\mathbf{z}, \mathbf{W}) & =\log p\left(\mathbf{y}_{\text {cat }} \mid \mathbf{z} ; \mathbf{W}\right) \\
L(\mathbf{z}, \mathbf{W}) & =\alpha \mathcal{L}_{1}(\mathbf{z}, \mathbf{W})+\mathcal{L}_{2}(\mathbf{z}, \mathbf{W})
\end{aligned}
$$

Where $\mathcal{L}_{1}$ is the loss function of the segmentation task with labels $\mathbf{y}_{\text {seg }}$. Similarly, $\mathcal{L}_{2}$ is the loss function of the categorization task with labels $\mathbf{y}_{\text {cat }}$. $L$ is the loss function that accounts for both tasks, where the segmentation task is weighted by an $\alpha$ scalar.

Stacked model. For this model, we use a multitask network as a feature extractor whose loss function is defined as a categorical cross entropy (see Figure 3). We apply a softmax activation function to produce the probability distribution over the labels, and then we calculate the loss as 
follows:

$$
\begin{aligned}
H_{1}(\mathbf{y}, \mathbf{z}) & =-\sum_{\mathbf{z}_{i}} \mathbf{y} \log \left(\operatorname{softmax}\left(\mathbf{W}_{\text {seg }} \mathbf{z}_{i}+\mathbf{b}\right)\right) \\
H_{2}(\mathbf{y}, \mathbf{z}) & =-\sum_{\mathbf{z}_{i}} \mathbf{y} \log \left(\operatorname{softmax}\left(\mathbf{W}_{\text {cat }} \mathbf{z}_{i}+\mathbf{b}\right)\right) \\
L(\mathbf{y}, \mathbf{z}) & =\alpha H_{1}\left(\mathbf{z}, \mathbf{W}_{\text {seg }}\right)+H_{2}\left(\mathbf{z}, \mathbf{W}_{\text {cat }}\right)
\end{aligned}
$$

After training the multitask network, we take the activation outputs from Equation 2. These vectors are used as features to train a Conditional Random Fields classifier. The definition of the CRF is the same as the one described for the end-to-end network.

\subsection{Implementation details}

We have performed a very simple preprocessing on the data, which consists in replacing URLs, emojis, tags, and numbers with predefined tokens. Additionally, the vocabulary of the pretrained word embeddings was not sufficient to cover all the words in the WNUT dataset (i.e., training, validation, and testing sets have OOV words). We handled this situation using the Facebook library FastText (Bojanowski et al., 2016). This library can produce an embedding vector from the subword level of the word (i.e., ngrams). The advantage of FastText over other embedding learning algorithms is that we can still extract useful embeddings for OOV words from their subword embeddings. For instance, if there is a missing letter in one word, the subword-level vector will be reasonably close to the vector of the correct spelling.

The models have been trained using weighted classes, which forces the models to pay more attention to the labels that are less frequent. This is a very important step since the NE datasets usually show a skewed distribution, where the NE tokens represent approximately $10 \%$ of the entire corpus. Although weighting classes improves the recall of the model, we tried to be sensitive to this aspect as the model can be forced to predict entities even in cases where there are none. The weights were experimentally defined, keeping the same distribution but decreasing the loss on non-entity tokens.

Additionally, we defined our models using the following hyperparameters: the phonetic and phonological BLSTM at the character level uses 64 units per direction, which adds up to 128 units. Similarly, the word level BLSTM uses 100 units per direction, which accounts for a total of 200

\begin{tabular}{llll}
\hline Corpus & Dataset & Classes & \% Unique \\
\hline \multirow{3}{*}{ CoNLL 2003 } & Train & 4 & $26 \%$ \\
& Dev & 4 & $40 \%$ \\
& Test & 4 & $41 \%$ \\
\hline \multirow{3}{*}{ WNUT 2017 } & Train & 6 & $75 \%$ \\
& Dev & 6 & $85 \%$ \\
& Test & 6 & $80 \%$ \\
\hline
\end{tabular}

Table 3: Percentage of unique NEs in two benchmark datasets, the one from CoNLL 2003 and the one used in the 2017 shared task held by the WNUT workshop.

units. The fully-connected layer has 100 neurons, and it uses a Rectified Linear Unit (ReLU) activation function. We also use a dropout operation before and after each BLSTM component. This forces the networks to find different paths to predict the data, which ultimately improves the generalization capabilities (i.e., they do not rely on a single path for certain inputs). The dropout value is 0.5. For the stacked model we use the Adam optimizer (Kingma and Ba, 2014) with a learning rate of 0.001 .

\section{Datasets}

Social media (SM) captures the fast evolving behavior of the language, and, as its influence in society grows, SM platforms play an important role in language understanding. We focus this work on the WNUT 2017 dataset for NER (Derczynski et al., 2017). This dataset covers multiple SM platforms and suits perfectly the purpose of this work. Table 5 shows the distribution of the dataset and its classes. The training set uses tweets, whereas the development set is based on YouTube comments. The testing set combines content from Reddit and StackExchange. The cross domain nature of the dataset establishes an additional challenge to the task. For instance, besides the particularities of the domains (e.g., length of the sentences, domainspecific expressions such as hashtags, emojis and others), the users tend to address different topics on each of the SM domains with different levels of relaxed language and style (Ritter et al., 2011; Strauss et al., 2016; Derczynski et al., 2017). Moreover, the predominant factors in those SM environments are the emerging and rare entities. As stated by Derczynski et al. (2017), emerging describes the entity instances that started to appear in context recently (e.g., a movie title released a 


\begin{tabular}{llll}
\hline Statistics & Train & Dev & Test \\
\hline Posts & 3,395 & 1,009 & 1,287 \\
Tokens & 62,729 & 15,733 & 23,394 \\
NE tokens & 3,160 & 1,250 & 1,589 \\
NE tokens (\%) & 5.04 & 7.95 & 6.79
\end{tabular}

Table 4: General statistics of the WNUT 2017 dataset. It is worth noting that the NE tokens account for less than $10 \%$ on any dataset, which shows the inherent skewness of the task.

\begin{tabular}{llll}
\hline Classes & Train & Dev & Test \\
\hline person & 995 & 46 & 532 \\
location & 793 & 238 & 188 \\
group & 414 & 64 & 202 \\
creative-work & 346 & 107 & 331 \\
product & 345 & 586 & 250 \\
corporation & 267 & 209 & 86 \\
\hline TOTAL & 3,160 & 1,250 & 1,589 \\
\hline
\end{tabular}

Table 5: Classes and their frequency distribution on the WNUT 2017 dataset.

year ago), whereas rare depicts the entities that appear less than certain number of times. It is worth noting that this dataset presents a great challenge to systems that rely on external resources due to the rare and emerging properties.

We also consider the CoNLL 2003 dataset (Tjong Kim Sang and De Meulder, 2003) as it has been used as the standard dataset for NER benchmarks. However, we emphasize that both datasets present significantly different challenges and, thus, some relevant aspects in CoNLL 2003 may not be that relevant in the WNUT 2017 dataset. For example, capitalization is a crucial feature in newswire text, but it is less important in SM data since users tend to arbitrarily alter the character casing. Moreover, the target classes on the WNUT 2017 dataset cover the CoNLL 2003 classes plus fine-grained classes such as creativework (e.g., movie titles, T.V. shows, etc.), group (e.g., sports teams, music bands, etc.), and product. The additional classes are more heterogeneous, and thus, it makes the task more difficult to generalize. Furthermore, Table 3 shows the percentage of unique tokens of the WNUT 2017 dataset, which certainly shows a great diversity compared to the CoNLL 2003 dataset.

\section{Experiments and results}

We mainly focus our experiments on the WNUT 2017 dataset. However, we consider relevant to compare our approach to the standard CoNLL 2003 dataset where current state-of-the-art systems are benchmarked. This section addresses the experiments and results of both datasets.

\subsection{WNUT 2017 experiments}

In this section we discuss the experiments of the proposed approaches. We compare our models and describe the contribution of each component of the stacked system. Additionally, we compare our results against the state of the art in the WNUT 2017 dataset.

Stacked vs. end-to-end model. Table 6 shows that the stacked system has a lower precision than the end-to-end model, but its recall is the highest. This means that the stacked model is slightly better at generalizing than the other models since it can detect a more diverse set of entities. The surface form F1 metric (Derczynski et al., 2017) supports that intuition as well. It assigns a better F1 score to the stacked system (43.90\%) than to the end-to-end model $(42.79 \%)$ because the former finds more rare and emerging entities than the latter. Moreover, Table 6 also shows that the precision of the end-to-end model is higher than the rest of the systems. This tends to capture the most frequent entities and leave behind the rare ones, which explains the different behaviors between the precision and recall of both models.

Stacked model. The feature extractor contains a category task that can produce predictions of the test set. We explored predicting the final labels with the feature extractor and compared the results against the predictions of the CRF classifier. We noticed that the CRF always outperformed the network. For the best scores the feature extractor achieved $40.64 \%$ whereas the CRF reached $45.55 \%$. This is consistent with previous research (Lample et al., 2016; Aguilar et al., 2017) in that the individual output probabilities of the network do not consider the whole sequence, and thus, a sequential algorithm such as a CRF can improve the results by learning global constraints (i.e., the B-person cannot be followed by I-corporation).

Ablation experiment. We explored the contribution of the features and different aspects of our models. For instance, we tried a BLSTM network using pretrained word embeddings only. The re- 


\begin{tabular}{llllllllll}
\hline \multirow{2}{*}{ Classes } & \multicolumn{3}{c}{ Precision (\%) } & \multicolumn{3}{c}{ Recall (\%) } & \multicolumn{3}{c}{ F1 (\%) } \\
& Stacked & E2E & WNUT & Stacked & E2E & WNUT & Stacked & E2E & WNUT \\
\hline corporation & 33.33 & 30.77 & 31.91 & 19.70 & 12.12 & 22.73 & 24.76 & 17.39 & 26.55 \\
creative-work & 50.00 & 55.56 & 36.67 & 14.79 & 10.56 & 7.75 & 22.83 & 17.75 & 12.79 \\
group & 47.76 & 63.16 & 41.79 & 19.39 & 14.55 & 16.97 & 27.59 & 23.65 & 24.14 \\
location & 62.20 & 78.12 & 56.92 & 52.67 & 50.00 & 49.33 & 57.04 & 60.98 & 52.86 \\
person & 73.49 & 71.15 & 70.72 & 51.05 & 51.75 & 50.12 & 60.25 & 59.92 & 58.66 \\
product & 40.58 & 34.29 & 30.77 & 22.05 & 9.45 & 9.45 & 28.57 & 14.81 & 14.46 \\
\hline Overall & 61.06 & $\mathbf{6 6 . 6 7}$ & 57.54 & $\mathbf{3 6 . 3 3}$ & 32.99 & 32.90 & $\mathbf{4 5 . 5 5}$ & 44.14 & 41.86 \\
\hline
\end{tabular}

Table 6: The class-level and overall results of our systems on the WNUT 2017 dataset. WNUT represents the winning system of the shared task (UH-RiTUAL), E2E is the end-to-end model, and Stacked shows the results of the stacked model. Both systems considerably outperform the state-of-the-art results. Between the end-to-end and the stacked models, the former gets better overall precision while the latter stands out on recall.

\begin{tabular}{lll}
\hline Model & F1 & Delta \\
\hline Stacked Model & $\mathbf{4 5 . 5 5}$ & \\
- Multitask Learning & 44.76 & -0.79 \\
- Character phonetics & 43.83 & -0.93 \\
- Weighted classes & 41.25 & -2.58 \\
- POS tag vectors & 40.15 & -1.10 \\
- FastText OOV vectors & 39.78 & -0.37 \\
- Pretrained embeddings & 12.72 & -27.06 \\
\hline
\end{tabular}

Table 7: We performed an ablation experiment on the stacked model. The results in the table are the average of the scores of three iterations.

sults of this model set our baseline on a $39.78 \%$ F1-score (see Table 7). This score is considerably close to the state-of-the-art performance, but improvements beyond that are small. For instance, Table 7 shows an ablation experiment using the stacked model. The ablation reveals that weighting the classes is the most influential factor, which accounts for a $2.58 \%$ of F1 score improvement. This aligns with the fact that the data is highly skewed, and thus, the model should pay more attention to the less frequent classes. The second most important aspect is the POS tags, which enhance the results by $1.10 \%$. This improvement suggests that POS tags are important whether the dataset is from a noisy environment or not since other researchers have found positive effects by using this feature on formal text (Huang et al., 2015). Almost equally influential are the phonetic and phonological features that push the F1 score by $0.93 \%$. According to the ablation experiment, using phonetic and phonology along with the pretrained word embeddings and POS tags can reach an F1 measure of $41.81 \%$, which is a very similar result to the state-of-the-art score, but with a simpler and more suitable model for SM environments (i.e., without gazetteers or capitalization).

We explored the multitask learning aspect by empirically trying multiple combinations of auxiliary tasks. The best combination is the standard NER categorization along with the segmentation task. The segmentation slightly improves the binary task proposed by Aguilar et al. (2017) by around $0.3 \%$. Additionally, trying the binarization, segmentation, and categorization tasks together drops the results by around $0.2 \%$ with respect to the categorization paired with the binary task. Moreover, the ablation experiment shows that the multitask layer boosts the performance of the stacked model with $0.79 \%$ of F1 score.

For the OOV problem, we use FastText to provide vectors to 2,333 words (around $13 \%$ of the vocabulary). However, the ablation experiment shows a small improvement, which suggests that those words did not substantially contribute to the meaning of the context. Another aspect that we explored was adding all the letters of the dataset to the character level of the stacked model without modifying the casing. Surprisingly, the models produced a slightly worse result (around $-0.5 \%$ ). Our intuition is that the character aspects are already captured by the model with the phonetic (IPA) representation, and the arbitrary use of capitalization renders this information useless. It is also worth noting that having phonetics instead of a language-dependent alphabet allows the adaptability of this approach to other languages.

State of the art comparison. Table 6 shows that our end-to-end and stacked models significantly outperform the state-of-the-art score by $2.28 \%$ and $3.69 \% \mathrm{~F} 1$ points, respectively. In the case of the stacked system, the precision and recall outper- 


\begin{tabular}{|l|l|}
\hline No & Predictions \\
\hline 1 & $\begin{array}{l}\text { Road and airport closure isolate Srinagar } \\
\text { as avalanche risk remains high }\end{array}$ \\
\hline 2 & $\begin{array}{l}\frac{\text { The Defence Research Development }}{\text { Organisation ( DRDO }) \text { is working on }} \\
\text { four projects to develop new technologies } \\
\text { for more accurate ... }\end{array}$ \\
\hline 3 & Her name is Scout . \\
\hline
\end{tabular}

Table 8: Examples of the predictions of our stacked model in the Reddit domain of the WNUT 2017 dataset. The bold words are the gold labels, and the underlined words are the predictions of our model. The model matches the entity types of the labeled data.

form the winning system of the shared task (UHRiTUAL) across all the classes. Moreover, even though the UH-RiTUAL system uses gazetteers, it only outperforms the recall of the end-to-end model on the corporation class. These results can be explained by the entity diversity of the dataset, where the emerging and rare properties are difficult to capture with external resources.

\subsection{CoNLL 2003 evaluation}

We also benchmarked our approach on a standard CoNLL 2003 dataset for the NER task. The stacked model reached $89.01 \%$ while the end-toend model achieved $88.98 \%$ on the F1 metric. Although the state-of-the-art performance is $91.21 \%$ (Ma and Hovy, 2016), our approach targets SM domains and, consequently, our models disregard some of the important aspects on formal text while still getting reasonable results. For instance, Ma and Hovy (2016) input the text to their model as $i s$, which indirectly introduce capitalization to the morphological analysis at the character level. This aspect becomes relevant in this dataset because entities are usually capitalized on formal text. As explained before, our models do not rely on capitalization because the characters are represented by the International Phonetic Alphabet, which does not differentiate between lower and upper cases.

\section{Analysis}

Table 8 shows some predictions of our stacked model on the WNUT 2017 test set. In example number 1, the model is able to correctly label Srinagar as person, even though the model does not rely on gazetteers or capitalization. It is also important to mention that the word was not in the training or development set, which means that the network had to infer the entity purely from the context. Moreover, the second example shows that the model has problems to determine whether the article the belongs to an NE or not. This is an ambiguous problem that even humans struggle with. This example also has a variation on spelling for the words Defence and Organisation. We suspect that the mitigation of OOV words using the FastText library helped in this case. Also, from the phonetic perspective, the model treated the word Defence as if it was the word Defense because both words map to the same IPA sequence, /difens/. In the third case, the model is not able to identify the NE Scout, even though the context makes it fairly easy.

\section{Related work}

In its former years, NER systems focused on newswire text, where the goal was to identify mainly three types of entities: person, corporation, and location. These entity types were originally proposed in the 6th Message Understanding Conference (MUC-6) (Grishman and Sundheim, 1996b). In MUC-7, the majority of the systems were based on heavily hand-crafted features and manually elaborated rules (Borthwick et al., 1998). Some years later, many researchers incorporated machine learning algorithms to their systems, but there was still a strong dependency on external resources and domain-specific features and rules (Tjong Kim Sang and De Meulder, 2003). In addition, the majority of the systems used Maximum Entropy (Bender et al., 2003; Chieu and Ng, 2003b; Curran and Clark, 2003; Florian et al., 2003b; Klein et al., 2003) and Hidden Markov Models (Florian et al., 2003b; Klein et al., 2003; Mayfield et al., 2003; Whitelaw and Patrick, 2003). Furthermore, McCallum and Li (2003) used a CRF combined with webaugmented lexicons. The features were selected by hand-crafted rules and refined based on their relevance to the domain of the entities. Moreover, Nothman et al. (2013) used Wikipedia resources to take advantage of structured data and reduce the human-annotated labels. In general, the results of the systems were reasonable for formal text, yet the scalability and the expensive detailed rules were not; their systems were difficult to maintain and adapt to other domains where different rules were needed. 
Recently, NER has been focused on noisy data as a result of the growth in social media users. However, the limits of the previous systems dramatically affected the results on noisy domains. For instance, Derczynski et al. (2014) evaluated multiple NER tools in noisy environments: Stanford NER (Finkel et al., 2005), ANNIE (Cunningham et al., 2002), among others. They reported that the majority of the tools were not capable of adapting to the noisy conditions showing a drop in performance of around $40 \%$ on a F1-score metric. This motivated many researchers to solve the problem using different techniques. In 2015, ? organized a NER shared task at the 1st Workshop on Noisy User-generated Text (WNUT), where three of the participants used word embedding as features to train their traditional machine learning algorithms (Godin et al., 2015; Toh et al., 2015; Cherry et al., 2015). The shared task introduced noisy data as well as more difficult entity types to identify (e.g., tv show, product, sports team, movie, music artist, etc.). Notably, the WNUT 2016 and 2017 were predominated by neural network systems (Limsopatham and Collier, 2016; Aguilar et al., 2017).

Deep neural networks have proven to be effective for NER. The state-of-the-art and the most competitive architectures can be characterized by the use of recurrent neural networks (Chiu and Nichols, 2016) combined with CRF (Lample et al., 2016; Ma and Hovy, 2016; Peng and Dredze, 2016; Bharadwaj et al., 2016; Aguilar et al., 2017). Our work primarily focuses on social media data and explores more suitable variations and combinations of those models. The most important differences of our approach and previous works are i) the use of phonetics and phonology (articulatory) features at the character level to model SM noise, ii) consistent BLSTMs for character and word levels, iii) the segmentation and categorization tasks, iv) a multitask neural network that transfers the learning without using lexicons or gazetteers, and v) weighted classes to handle the inherent skewness of the datasets.

\section{Conclusions}

This paper proposed two models for NER on social media environments. The first one is a stacked model that uses a multitask BLSTM network as a feature extractor to transfer the learning to a CRF classifier. The second one is an end-to-end multi- task BLSTM-CRF model whose output layer has a CRF per task. Both models improve the state-ofthe-art results on the WNUT 2017 dataset, where the data comes from multiple SM domains (i.e., Twitter, YouTube, Reddit, and StackExchange). Instead of working on normalizing text, we designed representations that are robust to inherent properties of SM data: inconsistent spellings, diverse vocabulary, and flexible grammar. Considering that SM is a prevalent communication channel that constantly generates massive amounts of data, it is practical to design NLP tools to process this domain as is. In this sense, we showed that the phonetic and phonological features are useful to capture sound-driven writing. This approach avoids the standard normalization process and boosts prediction performance. Furthermore, the use of multitask learning with segmentation and categorization is important to improve the results of the models. Finally, the weighted classes force the model to pay more attention on skewed datasets. We showed that these components can point to more suitable approaches for NER on social media data.

\section{References}

Gustavo Aguilar, Suraj Maharjan, Adrian Pastor López Monroy, and Thamar Solorio. 2017. A multitask approach for named entity recognition in social media data. In Proceedings of the EMNLP 3rd Workshop on Noisy User-generated Text. Association for Computational Linguistics, Copenhagen, Denmark, pages 148-153. http: //Www . aclweb.org/anthology/W17-4419.

Isabelle Augenstein, Leon Derczynski, and Kalina Bontcheva. 2017. Generalisation in named entity recognition: A quantitative analysis. CoRR abs/1701.02877. http://arxiv.org/abs/ 1701.02877.

Oliver Bender, Franz Josef Och, and Hermann Ney. 2003. Maximum entropy models for named entity recognition. In Walter Daelemans and Miles Osborne, editors, Proceedings of the Seventh Conference on Natural Language Learning at HLT-NAACL 2003. pages 148-151. http://www. aclweb. org/anthology/w03-0420.pdf.

Akash Bharadwaj, David Mortensen, Chris Dyer, and Jaime Carbonell. 2016. Phonologically aware neural model for named entity recognition in low resource transfer settings. In Proceedings of the 2016 Conference on Empirical Methods in Natural Language Processing. Association for Computational Linguistics, Austin, Texas, pages 1462 
1472. https://aclweb.org/anthology/ D16-1153.

Piotr Bojanowski, Edouard Grave, Armand Joulin, and Tomas Mikolov. 2016. Enriching word vectors with subword information. arXiv preprint arXiv:1607.04606.

A. Borthwick, J. Sterling, E. Agichtein, and R. Grishman. 1998. Nyu: Description of the mene named entity system as used in muc-7. In Seventh Message Understanding Conference (MUC-7): Proceedings of a Conference Held in Fairfax, Virginia, April 29 - May 1, 1998. http://www.aclweb.org/ anthology/M98-1018.

Colin Cherry, Hongyu Guo, and Chengbi Dai. 2015. Nrc: Infused phrase vectors for named entity recognition in twitter. In Proceedings of the Workshop on Noisy User-generated Text. Association for Computational Linguistics, Beijing, China, pages 5460. http: //www. aclweb.org/anthology/ W15-4307.

Hai Leong Chieu and Hwee Tou Ng. 2003a. Named entity recognition with a maximum entropy approach. In Proceedings of the Seventh Conference on Natural Language Learning at HLTNAACL 2003 - Volume 4. Association for Computational Linguistics, Stroudsburg, PA, USA, CONLL '03, pages 160-163. https://doi.org/10. $3115 / 1119176.1119199$.

Hai Leong Chieu and Hwee Tou Ng. 2003b. Named entity recognition with a maximum entropy approach. In Walter Daelemans and Miles Osborne, editors, Proceedings of the Seventh Conference on Natural Language Learning at HLT-NAACL 2003. pages 160-163. http://www.aclweb.org/ anthology/w03-0423.pdf.

Jason Chiu and Eric Nichols. 2016. Named entity recognition with bidirectional 1stmcnns. Transactions of the Association for Computational Linguistics 4:357-370. https://transacl.org/ojs/index. php/tacl/article/view/792.

Hamish Cunningham, Diana Maynard, Kalina Bontcheva, and Valentin Tablan. 2002. Gate: an architecture for development of robust hlt applications. In Proceedings of 40th Annual Meeting of the Association for Computational Linguistics. Association for Computational Linguistics, Philadelphia, Pennsylvania, USA, pages 168-175. https: //doi.org/10.3115/1073083.1073112.

James R. Curran and Stephen Clark. 2003. Language independent ner using a maximum entropy tagger. In Proceedings of the Seventh Conference on Natural Language Learning at HLTNAACL 2003 - Volume 4. Association for Computational Linguistics, Stroudsburg, PA, USA, CONLL '03, pages 164-167. https://doi.org/10. $3115 / 1119176.1119200$.
Leon Derczynski, Diana Maynard, Giuseppe Rizzo, Marieke van Erp, Genevieve Gorrell, Raphaël Troncy, Johann Petrak, and Kalina Bontcheva. 2014. Analysis of named entity recognition and linking for tweets. CoRR abs/1410.7182. http://arxiv. org/abs/1410.7182.

Leon Derczynski, Eric Nichols, Marieke van Erp, and Nut Limsopatham. 2017. Results of the WNUT2017 Shared Task on Novel and Emerging Entity Recognition. In Proceedings of the 3rd Workshop on Noisy, User-generated Text (WNUT) at EMNLP. ACL. http: / / aclweb.org/ anthology/W/W17/W17-4418.pdf.

Chris Dyer, Miguel Ballesteros, Wang Ling, Austin Matthews, and Noah A. Smith. 2015. Transitionbased dependeny parsing with stack long short-term memory. In Proceedings of the $53^{\text {rd }}$ Annual Meeting of the Association of Computational Linguistics and the $7^{\text {th }}$ International Joint Conference on Natural Language Processing of the Asian Federation of Natural Language Processing (ACL-IJCNLP 2015). ACL.

Jenny Rose Finkel, Trond Grenager, and Christopher Manning. 2005. Incorporating non-local information into information extraction systems by gibbs sampling. In Proceedings of the 43rd Annual Meeting on Association for Computational Linguistics. Association for Computational Linguistics, Stroudsburg, PA, USA, ACL '05, pages 363-370. https : //doi.org/10.3115/1219840.1219885.

Radu Florian, Abe Ittycheriah, Hongyan Jing, and Tong Zhang. 2003a. Named entity recognition through classifier combination. In Proceedings of the Seventh Conference on Natural Language Learning at HLT-NAACL 2003 - Volume 4. Association for Computational Linguistics, Stroudsburg, PA, USA, CONLL '03, pages 168-171. https: //doi.org/10.3115/1119176.1119201.

Radu Florian, Abe Ittycheriah, Hongyan Jing, and Tong Zhang. 2003b. Named entity recognition through classifier combination. In Walter Daelemans and Miles Osborne, editors, Proceedings of the Seventh Conference on Natural Language Learning at HLT-NAACL 2003. pages 168-171. http://www.aclweb.org/ anthology/W03-0425.pdf.

Fréderic Godin, Baptist Vandersmissen, Wesley De Neve, and Rik Van de Walle. 2015. Multimedia Lab @ ACL WNUT NER Shared Task: Named Entity Recognition for Twitter Microposts using Distributed Word Representations. In Proceedings of the Workshop on Noisy User-generated Text. Association for Computational Linguistics, Beijing, China, pages 146-153. http://www.aclweb.org/ anthology/W15-4322.

Ralph Grishman and Beth Sundheim. 1996a. Message understanding conference - 6: A brief history. In COLING-1996 Vol. 1. pages 466-471. 
Ralph Grishman and Beth Sundheim. 1996b. Message understanding conference-6: A brief history. In Proceedings of the 16th Conference on Computational Linguistics - Volume 1. Association for Computational Linguistics, Stroudsburg, PA, USA, COLING '96, pages 466-471. https : / / doi .org/ $10.3115 / 992628.992709$.

Sepp Hochreiter and Jürgen Schmidhuber. 1997. Long short-term memory. Neural Comput. 9(8):17351780. https://doi.org/10.1162/neco. 1997.9 .8 .1735 .

Zhiheng Huang, Wei Xu, and Kai Yu. 2015. Bidirectional LSTM-CRF models for sequence tagging. CoRR abs/1508.01991. http://arxiv.org/ $\mathrm{abs} / 1508.01991$.

Diederik P. Kingma and Jimmy Ba. 2014. Adam: A method for stochastic optimization. CoRR abs/1412.6980. http://arxiv.org/abs/ 1412.6980 .

Dan Klein, Joseph Smarr, Huy Nguyen, and Christopher D. Manning. 2003. Named entity recognition with character-level models. In Proceedings of the Seventh Conference on Natural Language Learning at HLT-NAACL 2003 - Volume 4. Association for Computational Linguistics, Stroudsburg, PA, USA, CONLL '03, pages 180-183. https://doi. org/10.3115/1119176.1119204.

John D. Lafferty, Andrew McCallum, and Fernando C. N. Pereira. 2001. Conditional random fields: Probabilistic models for segmenting and labeling sequence data. In Proceedings of the Eighteenth International Conference on Machine Learning. Morgan Kaufmann Publishers Inc., San Francisco, CA, USA, ICML '01, pages 282289. http://dl.acm.org/citation.cfm? $i d=645530.655813$.

Guillaume Lample, Miguel Ballesteros, Sandeep Subramanian, Kazuya Kawakami, and Chris Dyer. 2016. Neural architectures for named entity recognition. CoRR abs/1603.01360. http://arxiv.org/ abs/1603.01360.

Nut Limsopatham and Nigel Collier. 2016. Bidirectional lstm for named entity recognition in twitter messages. In Proceedings of the 2nd Workshop on Noisy User-generated Text (WNUT). The COLING 2016 Organizing Committee, Osaka, Japan, pages 145-152. http: / / aclweb.org/ anthology/W16-3920.

Xuezhe Ma and Eduard Hovy. 2016. End-toend sequence labeling via bi-directional lstm-cnnscrf. In Proceedings of the 54th Annual Meeting of the Association for Computational Linguistics (Volume 1: Long Papers). Association for Computational Linguistics, Berlin, Germany, pages 1064-1074. http: / / www. aclweb.org/ anthology/P16-1101.
James Mayfield, Paul McNamee, and Christine Piatko. 2003. Named entity recognition using hundreds of thousands of features. In Proceedings of the Seventh Conference on Natural Language Learning at HLTNAACL 2003 - Volume 4. Association for Computational Linguistics, Stroudsburg, PA, USA, CONLL '03, pages 184-187. https://doi.org/10. 3115/1119176.1119205.

Andrew McCallum and Wei Li. 2003. Early results for named entity recognition with conditional random fields, feature induction and web-enhanced lexicons. In Proceedings of the Seventh Conference on Natural Language Learning at HLTNAACL 2003 - Volume 4. Association for Computational Linguistics, Stroudsburg, PA, USA, CONLL '03, pages 188-191. https://doi.org/10. 3115/1119176.1119206.

David R. Mortensen, Patrick Littell, Akash Bharadwaj, Kartik Goyal, Chris Dyer, and Lori Levin. 2016. Panphon: A resource for mapping ipa segments to articulatory feature vectors. In Proceedings of COLING 2016, the 26th International Conference on Computational Linguistics: Technical Papers. The COLING 2016 Organizing Committee, Osaka, Japan, pages 3475-3484. http:// aclweb.org/anthology/C16-1328.

Joel Nothman, Nicky Ringland, Will Radford, Tara Murphy, and James R. Curran. 2013. Learning multilingual named entity recognition from wikipedia. Artif. Intell. 194:151-175. https://doi.org/ $10.1016 / j . a r t i n t .2012 .03 .006$.

Olutobi Owoputi, Brendan O'Connor, Chris Dyer, Kevin Gimpel, Nathan Schneider, and Noah A. Smith. 2013. Improved Part-of-Speech Tagging for Online Conversational Text with Word Clusters. In Proceedings of the 2013 Conference of the North American Chapter of the Association for Computational Linguistics: Human Language Technologies. Association for Computational Linguistics, Atlanta, Georgia, pages 380-390. http: / / www . aclweb.org/anthology/N13-1039.

Nanyun Peng and Mark Dredze. 2016. Improving named entity recognition for chinese social media with word segmentation representation learning. In Proceedings of the 54th Annual Meeting of the Association for Computational Linguistics (Volume 2: Short Papers). Association for Computational Linguistics, Berlin, Germany, pages 149-155. http: / / anthology . aclweb.org/P16-2025.

Alan Ritter, Sam Clark, Mausam, and Oren Etzioni. 2011. Named entity recognition in tweets: An experimental study. In Proceedings of the Conference on Empirical Methods in Natural Language Processing. Association for Computational Linguistics, Stroudsburg, PA, USA, EMNLP '11, pages 1524-1534. http://dl.acm.org/ citation.cfm?id=2145432.2145595. 
Benjamin Strauss, Bethany Toma, Alan Ritter, MarieCatherine de Marneffe, and Wei Xu. 2016. Results of the wnut16 named entity recognition shared task. In Proceedings of the 2nd Workshop on Noisy User-generated Text (WNUT). The COLING 2016 Organizing Committee, Osaka, Japan, pages 138-144. http://aclweb.org/ anthology/W16-3919.

Erik F. Tjong Kim Sang and Fien De Meulder. 2003. Introduction to the conll-2003 shared task: Language-independent named entity recognition. In Walter Daelemans and Miles Osborne, editors, Proceedings of the Seventh Conference on Natural Language Learning at HLT-NAACL 2003. pages 142-147. http://www.aclweb.org/ anthology/w03-0419.pdf.

Zhiqiang Toh, Bin Chen, and Jian Su. 2015. Improving twitter named entity recognition using word representations. In Proceedings of the Workshop on Noisy User-generated Text. Association for Computational Linguistics, Beijing, China, pages 141-145. http://www. aclweb.org/ anthology/w15-4321.

Casey Whitelaw and Jon Patrick. 2003. Named entity recognition using a character-based probabilistic approach. In Proceedings of the Seventh Conference on Natural Language Learning at HLTNAACL 2003 - Volume 4. Association for Computational Linguistics, Stroudsburg, PA, USA, CONLL '03, pages 196-199. https://doi.org/10. 3115/1119176.1119208.

Zhilin Yang, Ruslan Salakhutdinov, and William W. Cohen. 2016. Multi-task cross-lingual sequence tagging from scratch. CoRR abs/1603.06270. http: //arxiv.org/abs/1603.06270. 